\title{
WELL-POSEDNESS AND REGULARITY RESULTS FOR A DYNAMIC VON KÁRMÁN PLATE
}

\author{
M. E. BRADLEY \\ DEPART IENT OF MATHEMATICS \\ LNIVERSITY OF LOVISVILLE \\ LOTISIILLE, KY 40292 \\ (Received April 26, 1993 and in revised form September 20, 1994

\begin{abstract}
We consıder the problem of well-posedness and regularity of solutions for a dynamic von Kármán plate which is clamped along one portion of the boundary and which experiences boundary damping through "free edge" conditions on the remainder of the boundary We prove the existence of unique strong solutions for this system
\end{abstract}

Key Words. von Kármán plate, strong regularıty, well-posedness

AMS(MOS) subject classification. $35 \mathrm{~B} 65,4 i \mathrm{~N} 20,73 \mathrm{~K} 10$

1. INTRODUCTION. In this paper, we consider the well-posedness of the von Kármán system given by

$$
\begin{array}{rll}
w_{t t}-\gamma^{2} \Delta w_{t t}+\Delta^{2} w=[w, F(w)] & \text { in } & Q=\Omega \times(0, T) \\
w(0, \cdot)=w_{0} ; w_{t}(0, \cdot)=w_{1} & \text { in } & \Omega \\
w=\frac{\partial}{\partial \nu} w=0 & \text { on } & \Sigma_{0}=\Gamma_{0} \times(0, T) \\
\Delta w+(1-\mu) B_{1} w=-\frac{\partial}{\partial \nu} w_{t} & \text { on } & \Sigma_{1}=\Gamma_{1} \times(0, T) \\
\Delta w & & \\
\frac{\partial}{\partial \nu} \Delta w+(1-\mu) B_{2} w-\gamma^{2} \frac{\partial}{\partial \nu} w_{t t}=w_{t}-\frac{\partial^{2}}{\partial \tau^{2}} w_{t} & \text { on } & \Sigma_{1},
\end{array}
$$

where we assume $\Omega \subset \mathbf{R}^{2}$, with sufficiently smooth boundary $\Gamma=\Gamma_{0} \cup \Gamma_{1}$. Here, $0<\mu<\frac{1}{2}$ represents Poisson's ratio and the boundary operators $B_{1}$ and $B_{2}$ are given by

$$
\left.\begin{array}{l}
B_{1} w=2 n_{1} n_{2} w_{x y}-n_{1}^{2} w_{y y}-n_{2}^{2} w_{x x} \\
B_{2} w=\frac{\partial}{\partial \tau}\left[\left(n_{1}^{2}-n_{2}^{2}\right) w_{x y}+n_{1} n_{2}\left(w_{y y}-w_{x x}\right)\right]
\end{array}\right\}
$$

Also, $F(w)$ satisfies the system of equations

$$
\left.\begin{array}{l}
\Delta^{2} F=-[w, w] \\
F=\frac{\partial}{\partial \nu} F=0 \quad \text { on } \Sigma=\Gamma \times(0, \infty)
\end{array}\right\}
$$

where

$$
[\phi, \psi]=\frac{\partial^{2} \phi}{\partial x^{2}} \frac{\partial^{2} \psi}{\partial y^{2}}+\frac{\partial^{2} \phi}{\partial y^{2}} \frac{\partial^{2} \psi}{\partial x^{2}}-2 \frac{\partial^{2} \phi}{\partial x \partial y} \frac{\partial^{2} \psi}{\partial x \partial y} .
$$

The well-posedness and regularity of such a system is both a delicate and interesting problem. Such results are important in solving the problem of stabilization for system (1.1). Usual PDE techniques require the existence and uniqueness of "smooth" solutions to justify computations used in determining the stability and controllability of dynamical models. The stabilization of thin plates (and particularly the von Kármán system) is of current interest in the literature (see ([1], [2], [3], [4], [5])). The von Kármán nonlinearity poses many difficulties in obtaining the well-posedness and regularity results we seek. Difficulties also arise from the higher order boundary conditions on $\Sigma$. To handle these difficulties we adapt abstract results proven in [6] to our more difficult boundary conditions.

This paper will proceed as follows. In Section 2 we state the main results of our paper. After this we state the appropriate abstract results from [6] which will be useful in the proofs of our results. In Section 3 we prove the results stated in Section 2. 
2. STATEMENT OF RESULTS. Before stating the results we intend to prove, we define the meaning of "wcak solutions" through a variational equality. Let

$$
a(u, r)=\int_{s i}\left(\Delta w \Delta v+(1-\mu)\left(2 w_{x y} v_{x y}-u_{x x} v y y-w_{y y} v_{x x}\right)\right) d \Omega .
$$

We define the spaces

$$
H_{\Gamma_{0}}^{2}(\Omega)=\left\{w \in H^{2}(\Omega): w=\frac{\partial w}{\partial \nu}=0 \text { on } \Gamma_{0}\right\}
$$

with norm

$$
\|w\|_{H_{\Gamma_{0}}^{2}(\Omega)}^{2}=a(w, w)
$$

and

$$
H_{\Gamma_{0}}^{1}(\Omega)=\left\{w \in H^{1}(\Omega): w=0 \text { on } \Gamma_{0}\right\}
$$

with norm

$$
\|w\|_{H_{\Gamma_{0}}^{1}(\Omega)}^{2}=\int_{\Omega}\left(w^{2}+\gamma^{2}|\nabla u|^{2}\right) d \Omega
$$

We define the solution space $\mathcal{H}=H_{\Gamma_{0}}^{2}(\Omega) \times H_{\Gamma_{0}}^{1}(\Omega)$.

Definition 2.1. A function pair $\left(w, w_{t}\right) \in C((0 . T) ; \mathcal{H})$ is said to be a weak solution to system (1.1) if $\left(w(\cdot, 0), w_{t}(\cdot, 0)\right)=\left(w_{0}, w_{1}\right)$ and $w$ satisfies the variational equation

$$
\begin{array}{cc}
0=\left(w_{t t}, \varphi\right)+\gamma^{2}\left(\nabla w_{t t}, \nabla \varphi\right)+a(w, w)-([w, F], \varphi) & \\
+<w_{t}-\frac{\partial^{2} w_{t}}{\partial \tau^{2}}, \varphi>+<\frac{\partial w_{t}}{\partial \nu}, \frac{\partial \varphi}{\partial \nu}>\quad \forall \varphi \in H_{\Gamma_{0}}^{2}(\Omega),
\end{array}
$$

where here and throughout the paper $(\cdot, \cdot)$ denotes either the $L^{2}(\Omega)$-inner product or the duality pairing between $H_{\Gamma_{0}}^{2}(\Omega)$ and $\left[H_{\Gamma_{0}}^{2}(\Omega)\right]^{\prime}$, as is appropriate by context, and $\langle\cdot, \cdot\rangle$ represents the $L^{2}(\Gamma)$-inner product. We note that (2.1) holds in $H^{-1}[0, T]$.

ThEOREM 2.1. Given initial data $\left(w_{0}, w_{1}\right) \in \mathcal{H}$, there exists a unique weak solution to system (1.1), $\left(w, w_{t}\right) \in C([0, T), \mathcal{H})$ for any $T>0$.

Theorem 2.2. (Regularity): Assume in addition to Theorem 2.1 that the initial data satisfy

$$
\text { (ii) } \left.\begin{array}{l}
\Delta w_{0}+(1-\mu) B_{1} w_{0}=-\frac{\partial}{\partial \nu} w_{1} \\
\frac{\partial \Delta w_{0}}{\partial \nu}+(1-\mu) B_{2} w_{0}=w_{1}-\frac{\partial^{2}}{\partial \tau^{2}} w_{1}
\end{array}\right\} \quad \text { on } \Gamma_{1} .
$$

Then the unique solution to (1.1) has the regularity

$$
\begin{aligned}
\text { (i) } & \left(w, w_{t}\right) \in C\left((0, T) ;\left(H^{3}(\Omega) \cap H_{\Gamma_{0}}^{2}(\Omega)\right) \times H_{\Gamma_{0}}^{2}(\Omega)\right) \text {; } \\
\text { (ii) } & w_{t t} \in C\left((0, T) ; H_{\Gamma_{0}}^{1}(\Omega)\right) \\
\text { (iii) } & \text { equation (2.1) is satisfied for every } t \in[0, T) .
\end{aligned}
$$

ThEOREM 2.3. (Strong Regularity): In addition to Theorems 2.1 and 2.2 we assume that

$$
\text { (ii) } \left.\begin{array}{l}
\Delta w_{0}+(1-\mu) B_{1} w_{1}=-\frac{\partial w_{t t}(0)}{\partial \nu} \\
\frac{\partial \Delta w_{1}}{\partial \nu}+(1-\mu) B_{2} u_{1}^{\prime}=u_{t t}(0)-\frac{\partial^{2} u_{t}(0)}{\partial \tau^{2}}
\end{array}\right\} \quad \text { on } \Gamma_{1} \text {, }
$$


where $w_{t t}(0)$ is dericed from the equation (1.1). Then the unique solution guarantefl by Theorem 2.1 has the follouing regularity properties:

$$
\begin{aligned}
\text { (i) } & \left(w \cdot w_{t}\right) \in C\left((0, T):\left(I^{4}(\Omega) \cap I_{\Gamma_{0}}^{2}(\Omega)\right) \times\left(H^{3}(\Omega) \cap H_{\Gamma_{0}}^{2}(\Omega)\right)\right) \text {; } \\
\text { (ii) } & w_{t t} \in C\left((0, T): H_{\Gamma_{0}}^{2}(\Omega)\right) ; \\
\text { (iii) } & w_{t t t} \in C\left((0, T): H_{\Gamma_{0}}^{1}(\Omega)\right) .
\end{aligned}
$$

Moreover, equation (1.1) holds in the $L^{2}$-sense for cach $t \in[0, T]$.

The proofs of Theorems 2.1-2.3 will be based primarily on the work of Favini and Lasiecka [6]. That paper deals with abstract problems of the form

$$
\left\{\begin{array}{l}
M w_{t t}(t)+\mathcal{A} w(t)+\mathcal{A} G G^{*} \mathcal{A} w_{t}(t)+\mathcal{A} G f(w)(t)=\mathcal{F}\left(w^{\cdot}\right)(t) \\
w(t=0)=w_{0} ; \quad w_{t}(t=0)=w_{1}
\end{array}\right.
$$

which will be described in detail shortly. Our intention in this paper is to recast system (1.1) in the abstract framework of (2.4). We will then show that the results of [6] may be applied directly to or may be adapted for our system. For the purpose of self-containment, we now state the necessary background and results from [6] which will be useful in this present context.

Let $\mathcal{A}$ be a closed, positive self-adjoint operator on a Hilbert space $H$ with $\mathcal{D}(\mathcal{A}) \subset H$. Let $V$ be another (appropriately chosen) Hilbert space such that

$$
\mathcal{D}\left(\mathcal{A}^{1 / 2}\right) \subset V \subset H \subset V^{\prime} \subset\left[\mathcal{D}\left(\mathcal{A}^{1 / 2}\right)\right]^{\prime} .
$$

We assume that $M: V \rightarrow V^{\prime}$ is both bounded and boundedly invertible so that the restriction $\left.\widetilde{M} \equiv M\right|_{H}$ with domain $\mathcal{D}(\widetilde{M})=\{u \in V: M u \in H\}$ gives that $V=\mathcal{D}\left(\widetilde{M}^{1 / 2}\right)$.

The operator $G$ is defined on another Hilbert space, $U$. It is assumed that $G: U \rightarrow H$ is a bounded linear operator such that $G^{*} \mathcal{A} \in \mathcal{L}\left(\mathcal{D}\left(\mathcal{A}^{1 / 2}\right) ; H\right)$.

Finally, the nonlinear term $\mathcal{F}: \mathcal{D}\left(\mathcal{A}^{1 / 2}\right) \rightarrow V^{\prime}$ is assumed to be Frechét differentiable with derivative, denoted $D \mathcal{F}$, satisfying

$$
\|D \mathcal{F}(u) h\|_{V^{\prime}} \leq C\left(\|u\|_{\mathcal{D}\left(\mathcal{A}^{1 / 2}\right)}\right)\|h\|_{\mathcal{D}\left(\mathcal{A}^{1 / 2}\right)} .
$$

We note that for our purposes, $f \equiv 0$.

We now state the results from [6] which form the framework for Theorem 2.1-2.3.

TheOREм 2.4. (F-L Theorem 2.1): For each initial data $\left(w_{0}, w_{1}\right) \in \mathcal{D}\left(\mathcal{A}^{1 / 2}\right) \times V$, there exists $T_{0}>0$ such that there exists a unique weak solution $\left(w(t), w_{t}(t)\right)$ to (2.4).

THEOREM 2.5. (F-L Theorem 2.4): In addition to the hypotheses of Theorem 2.4 we assume that for all $\tilde{w}=\left(w, w_{t}\right) \in C\left(0, T_{0} ; \mathcal{D}\left(\mathcal{A}^{1 / 2}\right) \times V\right)$ and such that $G^{*} \mathcal{A} w_{t} \in L^{2}\left(0, T_{0} ; U\right)$ the following inequality holds for all $t \in\left[0, T_{0}\right)$ :

$$
\begin{aligned}
\int_{0}^{t}\left(\mathcal{F}(w(\tau)), w_{t}(\tau)\right) d \tau & \leq C_{1} \int_{0}^{t}\left(\|w(\tau)\|_{\mathcal{D}\left(\mathcal{A}^{1 / 2}\right)}^{2}+\left\|w_{t}(\tau)\right\|_{V}^{2}\right) d \tau \\
& +C_{2}\left(\left\|\left(w_{0}, w_{1}\right)\right\|_{\mathcal{D}\left(\mathcal{A}^{1 / 2}\right) \times V^{2}}\right) \equiv C_{0}
\end{aligned}
$$

Then the weak solution $\left(w(t), w_{t}(t)\right)$ is global for any $T>0$.

Theorem 2.6. (F-L Theorem 2.2): Assume that the initial data $\left(w_{0}, w_{1}\right)$ satisfy

(i) $\quad w_{1} \in \mathcal{D}\left(\mathcal{A}^{1 / 2}\right)$

(ii) $\quad \mathcal{A}\left(w_{0}+\beta G G^{*} \mathcal{A} w_{1}\right) \in V^{\prime}$.

Moreover, assume that

$$
\left\|\mathcal{A}^{-1 / 2} D \mathcal{F}(w) h\right\|_{H} \leq C\left(\|w\|_{\mathcal{D}\left(\mathcal{A}^{1 / 2}\right)}\right)\|h\|_{V}
$$




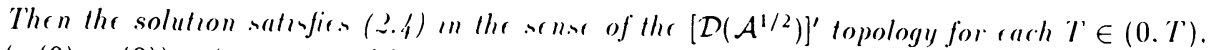
$\left(u \cdot(0), w_{t}(0)\right)=\left(u_{0}, u_{1}\right)$ and has the follow'mg regularity:

$$
\begin{aligned}
& \left(u, u_{t}\right) \in\left({ }^{\prime}\left(0 . T: \mathcal{D}\left(\mathcal{A}^{1 / 2}\right) \times \mathcal{D}\left(\mathcal{A}^{1 / 2}\right)\right) .\right. \\
& u_{t t} \in\left(^{\prime}(0 . T: 1) .\right.
\end{aligned}
$$

By showing that system (1.1) can be formulated in the framework of the almatrat equation (2.t) while satisfying the hypotheses of Theorems 2.5 2.7. we will have proven Theorems 2.1 and 22 . For the additional regularity given in Theorem 2.3, we wiil need an additional proof which does not follow directly from results of $[6]$.

3. PROOFS OF THEOREMS 2.1-2.3. Let $I=H_{\Gamma_{0}}^{1}(\Omega), H=L^{2}(\Omega)$ and $U^{\prime}=\left(L^{2}\left(\Gamma_{1}\right)\right)^{3}$. We define $\mathcal{A}$ on $H_{\Gamma_{0}}^{2}(\Omega)$ by

$$
\begin{aligned}
\mathcal{A} w \equiv & \Delta^{2} w \text { with domain } \\
D(\mathcal{A})= & \left\{w \in H^{4}(\Omega) \cap H_{\Gamma_{0}}^{2}(\Omega): \Delta w+(1-\mu) B_{1} u=0\right. \\
& \text { and } \left.\frac{\partial}{\partial \nu} \Delta w+(1-\mu) B_{2} w=0 \text { on } \Gamma_{1}\right\},
\end{aligned}
$$

which is well-defined, positive and self-adjoint. By the results of Grisvard [7], we see that $\mathcal{D}\left(\mathcal{A}^{1 / 2}\right)=$ $H_{\Gamma_{0}}^{2}(\Omega)$. We also define the Green maps, $G_{1}: H^{s}(\Gamma) \rightarrow H^{5 / 2+s}(\Omega), G_{2}: H^{s}(\Gamma) \rightarrow H^{\tau / 2+s}(\Omega)$ and $G_{3}: H^{s}(\Gamma) \rightarrow H^{5 / 2+s}(\Omega)$ by

$$
\begin{array}{cc}
G_{1} h=v \Longleftrightarrow \begin{array}{ll}
\Delta^{2} v=0 \\
v=\frac{\partial}{\partial \nu} v=0 \\
\Delta v+(1-\mu) B_{1} v=h \\
\frac{\partial}{\partial \nu} \Delta v+(1-\mu) B_{2} v=0
\end{array} & \text { in } Q \\
& \text { on } \sum_{0} \\
\left.G_{2} h=v \Longleftrightarrow \begin{array}{l}
\Delta^{2} v=0 \\
v=\frac{\partial}{\partial \nu} v=0 \\
\Delta v+(1-\mu) B_{1} v=0 \\
\frac{\partial}{\partial \nu} \Delta v+(1-\mu) B_{2} v=h
\end{array}\right\} & \text { on } \sum_{1}, \\
& \text { in } Q \\
& \text { on } \sum_{0}
\end{array}
$$

and

$$
G_{3} h=G_{2} \frac{\partial h}{\partial \tau}
$$

A straightforward computation shows that for $w \in \mathcal{D}(\mathcal{A})$,

$$
\begin{aligned}
G_{1}^{*} \mathcal{A} w & =\left.\frac{\partial w}{\partial \nu}\right|_{\Gamma_{1}} \\
G_{2}^{*} \mathcal{A} w & =-\left.w\right|_{\Gamma_{1}} . \\
G_{3}^{*} \mathcal{A} w & =\left.\frac{\partial w}{\partial \tau}\right|_{\Gamma_{1}} .
\end{aligned}
$$

Let $\bar{u} \in\left[L^{2}(\Gamma)\right]^{3}$. Define $G \bar{u}=-G_{1} u_{1}-G_{2} u_{2}-G_{3}\left(\frac{\partial u_{3}}{\partial \tau}\right)$. Then $G:\left[L^{2}(\Gamma)\right]^{3} \rightarrow L^{2}(\Omega)$ is bounded and $G^{*} \mathcal{A} \in \mathcal{L}\left(H_{\Gamma_{0}}^{2}(\Omega) ;\left[L^{2}(\Gamma)\right]^{3}\right)$.

We now introduce the operator $M: \mathcal{D}(M) \subset H^{2}(\Omega) \rightarrow L^{2}(\Omega)$.

$$
M w=\left(I-\gamma^{2} \Delta\right) w+\gamma^{2} \mathcal{A} G_{2} \frac{\partial w}{\partial \nu} .
$$

We observe that for $v, w \in H_{\Gamma_{0}}^{1}(\Omega)$,

$$
\begin{aligned}
(M w, v)= & (w, v)+\gamma^{2}(\nabla w, \nabla v) \\
& +\gamma^{2}\left\langle\frac{\partial w}{\partial \nu}, G_{2}^{*} \mathcal{A} v^{v}\right\rangle-\gamma^{2}\left\langle\frac{\partial w}{\partial \nu}, v\right\rangle \\
= & \left(v, w^{v}\right)+\gamma^{2}\left(\nabla v, \nabla w^{\prime}\right),
\end{aligned}
$$


where we have interpreted the $\gamma^{2} \mathcal{A} G_{2} \frac{\partial w}{\partial \nu}$ term in the sense of duality. I'sing (3.5). we see that $M: H_{\Gamma_{0}}^{1}(\Omega) \rightarrow\left[H_{\Gamma_{0}}^{1}(\Omega)\right]^{\prime}$ is an isomorphism (by the Lax-Milgramı Theorem).

By a straightforward computation, we see that

$$
(A u \cdot \varphi)=a(u, \varphi)
$$

and that

$$
\left\langle G^{*} A w_{t}, G^{*} A \varphi\right\rangle=\left\langle w_{t}-\frac{\partial^{2} u_{t}}{\partial \tau^{2}}, \varphi\right\rangle+\left\langle\frac{\partial u_{t}}{\partial \nu}, \frac{\partial \varphi}{\partial \nu}\right\rangle
$$

Defining $\mathcal{F}(w)=[w, F(w)]$ and using (3.6) - (3.8), we can now rewrite system (1.1) in the form of (2.4).

To see that the von Kármán nonlinearity is Frechét differentiable, we define the operator

$$
A_{0} w=\Delta^{2} w \text { with } \mathcal{D}\left(A_{0}\right)=H^{4}(\Omega) \cap H_{0}^{2}(\Omega) .
$$

Then $F(w)=-A_{0}^{-1}[w, w]$ so that $\mathcal{F}(w)=-\left[w, A_{0}^{-1}[u, w]\right]$. By straightforward (but somewhat lengthy) computations we see that

$$
D \mathcal{F}(w) h=\left[h, A_{0}^{-1}[w, w]\right]+2\left[w \cdot A_{0}^{-1}[w, h]\right] .
$$

To prove that $\|D \mathcal{F}(w) h\|_{\left[H_{\Gamma_{0}}^{1}(\Omega)\right]^{\prime}} \leq C\left(\|w\|_{H_{\Gamma_{0}}^{2}(\Omega)}\right)\|h\|_{H_{\Gamma_{0}}^{2}(\Omega)}$, we use the following lemma, which is proved in [3].

Lemma 3.1. The mapping $(u, v, w) \rightarrow\left[u, A_{0}^{-1}[v, w]\right]$ is continuous from $\left[H^{2}(\Omega)\right]^{3} \rightarrow H^{-\varepsilon}(\Omega)$ for $0<\varepsilon<1 / 2$.

Consequently, we have

$$
\|D \mathcal{F}(w) h\|_{\left[H_{\Gamma_{0}}^{1}(\Omega)\right]^{\prime}} \leq\|D \mathcal{F}(w) h\|_{H^{-\epsilon}(\Omega)} \leq C\|w\|_{H_{\Gamma_{0}}^{2}(\Omega)}^{2}\|h\|_{H_{\Gamma_{0}}^{2}(\Omega)} .
$$

Remark. An interesting estimate which arises in the proof of Lemma 3.1 is

$$
\left\|A_{0}^{-1}[w, v]\right\|_{H^{3-\varepsilon}(\Omega)} \leq C\|w\|_{H^{2}(\Omega)}\|v\|_{H^{2}(\Omega)} .
$$

This will be useful to us later in the proof.

PROOF OF THEOREM 2.1. To complete the proof, it suffices to show that (2.5) holds. Let $\left(w, w_{t}\right) \in C\left([0, T] ; H_{\Gamma_{0}}^{2}(\Omega) \times H_{\Gamma_{0}}^{1}(\Omega)\right)$. Then

$$
\begin{aligned}
& \int_{0}^{t} \int_{\Omega}[w, F(w)] w_{t} d \Omega d t=\int_{0}^{t} \int_{\Omega}\left[w, w_{t}\right] F(w) d \Omega d t \\
& \quad=\int_{0}^{t} \int_{\Omega} \frac{1}{2}\left(\frac{d}{d t}[w, w]\right) F(w) d \Omega d t \\
& \quad=-\frac{1}{2} \int_{0}^{t} \int_{\Omega} \frac{d}{d t}\left(\Delta^{2} F(w)\right) F(w) d \Omega d t \\
& \quad=-\frac{1}{4} \int_{0}^{t} \int_{\Omega} \frac{d}{d t}(\Delta F(w))^{2} d \Omega d t \\
& \quad \leq \frac{1}{4} \int_{\Omega}\left(\Delta F\left(w_{0}\right)\right)^{2} d \Omega=C\left\|F\left(w_{0}\right)\right\|_{H^{2}(\Omega)}^{2} \\
& \quad \leq C\left\|w_{0}\right\|_{H^{2}(\Omega)}^{2}
\end{aligned}
$$

where the last inequality holds by (3.11). Hence, (2.5) holds with $C_{1} \equiv 0$.

PROOF OF THEOREM 2.2. It suffices to verify (2.6) and (2.7) and to apply Theorem 2.6. We note that (2.6)(i) is satisfied by hypothesis (2.2)(i) in Theorem 2.2. As for (2.6)(ii), we see that in p.d.e. form this is equivalent to

$$
\begin{aligned}
& \Delta^{2} w_{0} \in\left[H_{\Gamma_{0}}^{1}(\Omega)\right]^{\prime} \\
& w_{0}=\frac{\partial w_{0}}{\partial \nu}=0 \text { on } \Gamma_{0} \\
& \left.\begin{array}{l}
\Delta w_{0}+(1-\mu) B_{1} w_{0}=-\frac{\partial w_{1}}{\partial \nu} \\
\frac{\partial \Delta w_{0}}{\partial \nu}+(1-\mu) B_{2} w_{0}=w_{1}-\frac{\partial^{2} w_{1}}{\partial \tau^{2}}
\end{array}\right\} \text { on } \Gamma_{1} .
\end{aligned}
$$

But then if $w_{0} \in H^{3}(\Omega) \cap H_{\Gamma_{0}}^{2}(\Omega)$ and $\left(w_{0}, w_{1}\right)$ satisfy the compatibility relation (2.2)(ii), we see that (2.6) must hold also. 
We now prove (2. $\tau$ ). We need to show that for $u \in H_{l_{0}}^{2}(\Omega), h \in H_{1_{0}}^{1}(\Omega)$ and $\rho \in H_{I_{0}}^{2}(\Omega)$ that

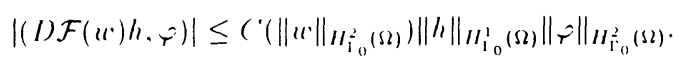

Recalling (3.9)-(3.10). we compute

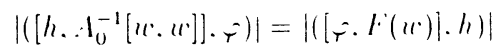

$$
\begin{aligned}
& \leq\|h\|_{U_{1_{0}}^{\prime}(s)} \|\left[\hat{r} \cdot F(u(c)] \|_{\left[H_{1_{1}}^{\prime}(s)\right]^{\prime}}\right. \\
& \leq\|h\|_{H_{\Gamma_{0}^{1}}^{1}(\Omega)} \|\left[\vartheta \cdot A_{0}^{-1}[u \cdot u] \|_{H^{-s(\Omega)}}\right. \\
& \leq C^{\prime}\left(\|w\|_{H_{I_{0}(\Omega)}^{2}(\Omega)}^{2}\right)\|h\|_{H_{I_{0}}^{1}(\Omega)}\|\vartheta\|_{H_{1_{0}}^{2}(\Omega)} \text {. }
\end{aligned}
$$

where we have used Lemma 3.1.

We now compute

$$
\begin{aligned}
& \left|\left(\left[u, A_{0}^{-1}[u, h]\right] \cdot \vartheta\right)\right|=\left|\left([u \cdot \vartheta], A_{0}^{-1}[u \cdot h]\right)\right| \\
& \quad \leq\|[w, \varphi]\|_{H^{-1-\varepsilon}(\Omega)}\left\|A_{0}^{-1}[u, h]\right\|_{H_{0}^{1+\varepsilon}(\Omega)} \\
& \quad \leq C\|w\|_{H_{\Gamma_{0}}^{2}(\Omega)}\|\varphi\|_{H^{2}(\Omega)}\left\|A_{0}^{-\left(\frac{3}{4}\right)+\varepsilon / 4}[w \cdot h]\right\|_{L^{2}(\Omega)} .
\end{aligned}
$$

where we have again used the results of Grisiard [i] to give us $\mathcal{D}\left(A_{0}^{\frac{1+\varepsilon}{4}}\right) \sim I_{0}^{1+\varepsilon}(\Omega)$.

We now examine the term $\left\|A_{0}^{-3 / 4+s / 4}[u, h]\right\|_{L^{2}(\Omega)}$. Let $v^{\prime} \in \mathcal{D}\left(A_{0}^{3 / 4-\varepsilon / 4}\right)$ so that (again by Grisvard's results) we have $\psi \in H^{3-\varepsilon}(\Omega) \cap H_{0}^{2}(\Omega)$. Then

$$
\left|\left([w, h], \psi^{\prime}\right)\right|=\left|\left(\left[w, \psi^{\prime}\right], h\right)\right| \leq C^{\prime}\left\|w^{\prime}\right\|_{H^{2}(\Omega)}\left(\int_{\Omega}\left(\psi_{y y}^{2}+\psi_{x x}^{2}+u_{x y}^{2}\right) h^{2} d \Omega\right)^{1 / 2}
$$

But then since $h \in H^{1}(\Omega) \subset L^{q}(\Omega), 1 \leq q<\infty$, and by Hölder's inequality, we have, for example,

$$
\begin{aligned}
\int_{\Omega} \psi_{y y}^{2} h^{2} d \Omega & \leq\left(\int_{\Omega} \psi_{y y}^{2 p} d \Omega\right)^{1 / p}\left(\int_{\Omega} h^{2 q} d \Omega\right)^{1 / q} \\
& =\left\|\psi_{y y}\right\|_{L^{2 p}(\Omega)}^{2}\|h\|_{L^{2 q}(\Omega)}^{2} \\
& \leq \bar{C}\left\|\psi_{y y}\right\|_{L^{2+c_{0}(\Omega)}}^{2}\|h\|_{H^{1}(\Omega)}^{2} .
\end{aligned}
$$

Using the Sobolev imbeddings (see [8], Theorem 7.58 p. 218), this implies

$$
\left\|\psi_{y y} h\right\|_{L^{2}(\Omega)}^{2} \leq C\|\psi\|_{H^{2+c_{1}(\Omega)}}^{2}\|h\|_{H^{1}(\Omega)}^{2},
$$

where $\varepsilon_{1}=\frac{\varepsilon_{0}}{2+\varepsilon_{0}}$. Substituting back into (3.14), we obtain

$$
\begin{aligned}
|([w, \psi], h)| & \leq \tilde{C}\|w\|_{H^{2}(\Omega)}\|\psi\|_{H^{2+c}(\Omega)}\|h\|_{H^{1}(\Omega)} \\
& \leq C\|w\|_{H^{2}(\Omega)}\|\psi\|_{H^{3-\varepsilon}(\Omega)}\|h\|_{H^{1}(\Omega)} .
\end{aligned}
$$

Putting (3.13)-(3.15) together implies

$$
\left|\left(\left[w, A_{0}^{-1}[w, h]\right], \varphi\right)\right| \leq C\|w\|_{H_{\Gamma_{0}}^{2}(\Omega)}^{2}\|h\|_{H_{\Gamma_{0}}^{1}(\Omega)}^{2}\|\varphi\|_{H_{\Gamma_{0}}^{2}(\Omega)} .
$$

Then taking (3.12) with (3.16) gives us the estimate in (2.7). Applying Theorem 2.6, we have the result.

PROOF OF THEOREM 2.3. Here we would like to use the following strong regularity result from [6].

TheOREM 3.2. ([6] Theorem 2.3 - Regularity Revisited): In addition to the assumptions of the previous theorem (our Theorem 2.6) assume that $\mathcal{F}$ is twice Frechét differentiable $\mathcal{D}\left(\mathcal{A}^{1 / 2}\right) \rightarrow V^{\prime}$. Moreover, assume

$$
\widetilde{M}^{-1} \in \mathcal{L}\left(H ; \mathcal{D}\left(\mathcal{A}^{1 / 2}\right)\right)
$$




$$
\mathcal{F}\left(w_{u}\right) \in I I:
$$

and

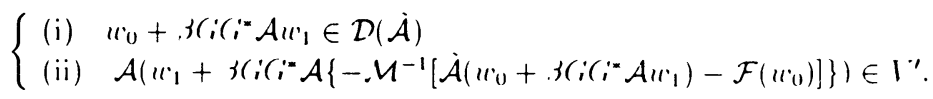

Then.

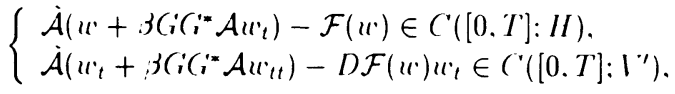

and the equation

$$
.1 / u_{t t}+\mathcal{A}\left(u^{\prime}(t)+, 3\left(i_{i}\left(i^{*} \mathcal{A} w_{t}(t)\right)-\mathcal{F}\left(w^{\prime}(t)\right)=0\right.\right.
$$

holds for all $t \geq 0$ on $I I$.

Unfortunately, system (1.1) fails to satisfy hypothesis (3.17), since for general $L^{2}$-functions, $\widetilde{\mathcal{M}}^{-1}$ cannot recover both boundary conditions on $\Gamma_{0}$. However, to follow the proof of the theorem given in [6], we need only

$$
M^{-1} \mathcal{A}\left(w_{0}+\beta G G^{*} \mathcal{A} w_{1}\right)+M^{-1} \mathcal{F}\left(w_{0}\right) \in \mathcal{D}\left(\mathcal{A}^{1 / 2}\right),
$$

which, in terms of system (1.1) requires $w_{t t}(0) \in \mathcal{D}\left(\mathcal{A}^{1 / 2}\right)$. By virtue of hypothesis on $w_{0}, w \in$ $\mathcal{D}\left(\mathcal{A}^{1 / 2}\right)$, it suffices that $\widetilde{M}^{-1}: L^{2}(\Omega) \rightarrow H^{2}(\Omega)$. But this follows directly from the definition of $\widetilde{M}$. Consequently, system (1.1) satisfies the weaker, but sufficient, hypothesis (3.23). We now show that under the hypotheses of Theorem 2.3, we may apply the modified version of Theorem 3.1 to system (1.1).

By straightforward computations one can see that the von Kármán nonlinearity is twice Frechét differentiable with

$$
\begin{aligned}
D^{2} \mathcal{F}(w)(h, v) & =\left[-2 A_{0}^{-1}[w, h], v\right] \\
& +\left[-2 A_{0}^{-1}[v, h], w\right]+\left[h,-2 A_{0}^{-1}[w, v]\right] .
\end{aligned}
$$

By Lemma 3.1 we see that for $w, h, v \in H_{\Gamma_{0}}^{2}(\Omega)$ with $\varepsilon<1 / 2$,

$$
\begin{aligned}
\left\|D^{2} \mathcal{F}(w)(h, v)\right\|_{\left[H_{\Gamma_{0}}^{1}(\Omega)\right]^{\prime}} & \leq\left\|D^{2} \mathcal{F}(w)(h, v)\right\|_{H^{-\varepsilon}(\Omega)} \\
& \leq C\|w\|_{H_{\Gamma_{0}}^{2}(\Omega)}\|h\|_{H_{\Gamma_{0}}^{2}(\Omega)}\|v\|_{H_{\Gamma_{0}}^{2}(\Omega)} .
\end{aligned}
$$

By hypothesis $(2.3)(\mathrm{i})$, we see that $\mathcal{F}\left(w_{0}\right) \in L^{2}(\Omega)$ is trivially satisfied. $(2.4)$

In terms of the p.d.e., (3.19)(i) is equivalent to (2.3)(i) with (2.2)(ii). We also observe that by

$$
-\mathcal{M}^{-1}\left[\mathcal{A}\left(w_{0}+\beta G G^{*} \mathcal{A} w_{1}\right)-\mathcal{F}\left(w_{0}\right)\right]=w_{t t}(0),
$$

So that the p.d.e. equivalent of (3.19)(ii) is

$$
\begin{aligned}
& \Delta^{2} w_{1} \in\left[H_{\Gamma_{0}}^{1}(\Omega)\right]^{\prime} \\
& w_{1}=\frac{\partial w_{1}}{\partial \nu}=0 \text { on } \Gamma_{0} \\
& \left.\begin{array}{l}
\Delta w_{1}+(1-\mu) B_{1} w_{1}=-\frac{\partial}{\partial \nu} w_{t t}(0) \\
\frac{\partial \Delta w_{1}}{\partial \nu}+(1-\mu) B_{2} w_{1}=w_{t t}-\frac{\partial^{2}}{\partial \tau^{2}} w_{t t}(0)
\end{array}\right\} \quad \text { on } \Gamma_{1} .
\end{aligned}
$$

But these are precisely satisfied by hypothesis (2.3)

Applying the results of Theorem 3.1, we obtain the regularity results of Theorem 2.3 . 
4. ACKNOWLEDGEMENT. This work was completed while the author was at the Institue for Mathematics and its Applications at the University of Minnesota. This visit to the IMA was sponsored in part by a grant from the Iniversity of Louisville.

\section{REFERENCES}

[1] Lagnese, J. Boundary Stabilization of Thın Plates SIAM, Plıladelphiı. 1989!

[2] Lagnese, J Local Controllability of dynamic ron fiarmán plates ('ontrol and ('vberuetics. vol 19. no's 3-4, pp) $155-168,1990$

[3] Bradley, M.E. and I. Lasiecka. Local Stabulzatıon for a Nonlınearly Perturbed von Kármán Plate. Nonlınear Analysis: Theory, Methods and Applications, vol. 18, no 4, pp. 333-343, 1992.

[4] Puél, J. and M. Tucsiak. Boundary stabilization for the von Kármán equations to appear in SIAM J. on Control and Optimization.

[5] Lagnese, J. and J.-L. Lions. Modelling Analysis and Control of Thm Plates. Masson, Paris, 1988.

[6] Favini, A. and I. Lasiecka. W' $\epsilon$ ll-posedness and regularty if second order abstract equatıons arising in hyperboliclike problems with nonlinear boundary conditions. Preprint.

[7] Grisvard, P. Elliptıc Problems in Nonsmooth Domaıns. Pitman, London, 1985.

[8] Adams, R. Sobolev Spaces. Academic Press, New York, 1975. 


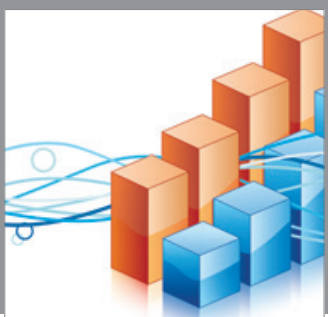

Advances in

Operations Research

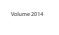

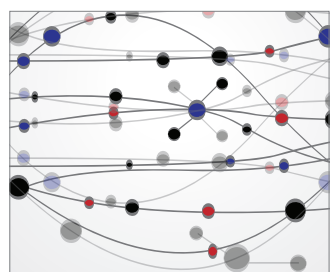

\section{The Scientific} World Journal
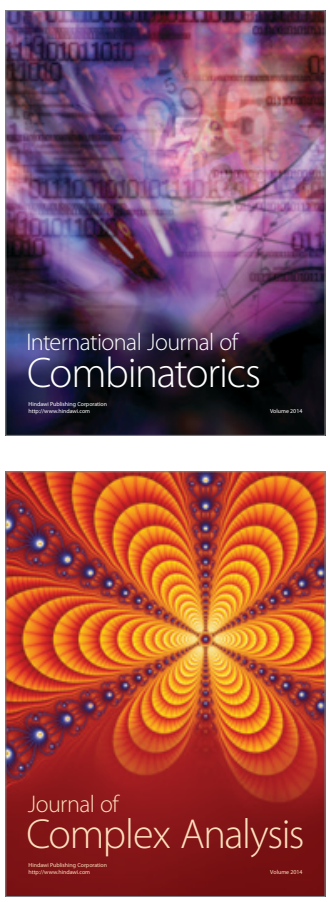

International Journal of

Mathematics and

Mathematical

Sciences
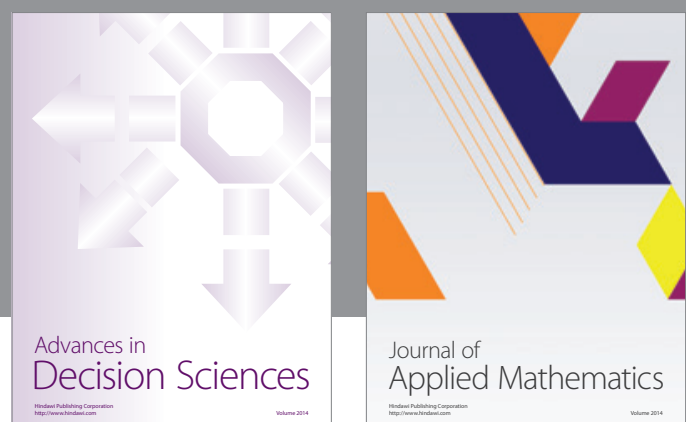

Journal of

Applied Mathematics
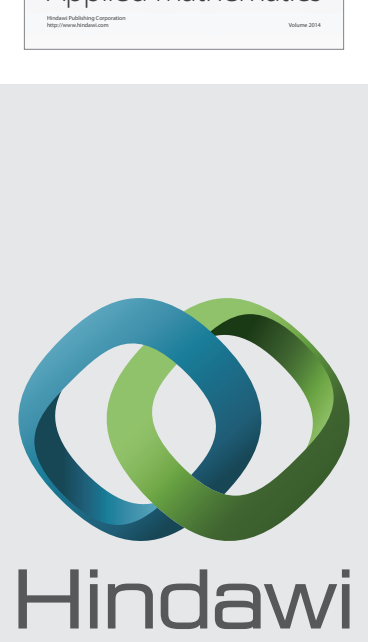

Submit your manuscripts at http://www.hindawi.com
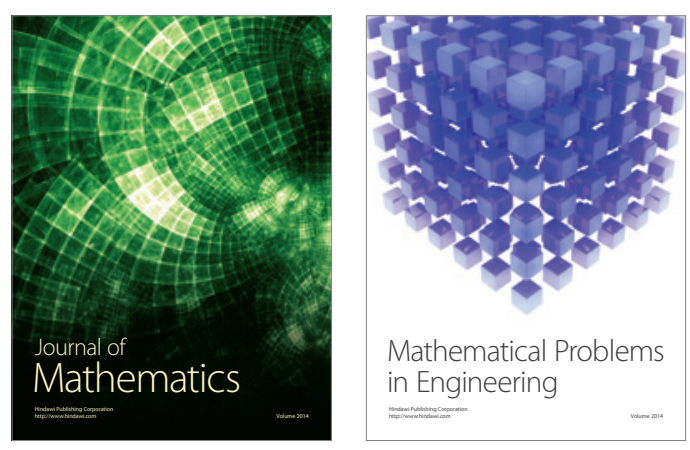

Mathematical Problems in Engineering
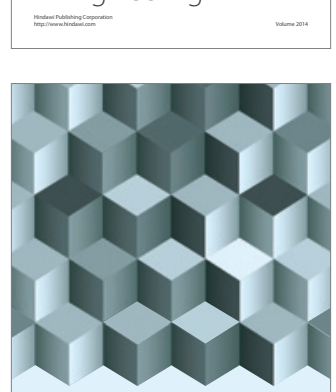

Journal of

Function Spaces
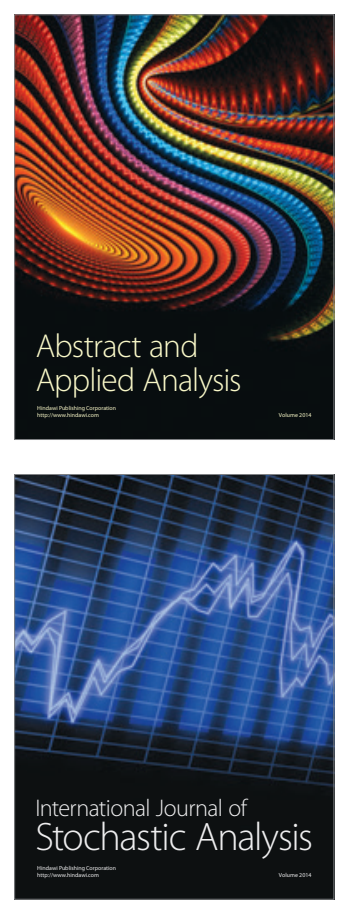

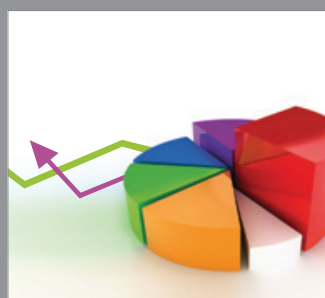

ournal of

Probability and Statistics

Promensencen
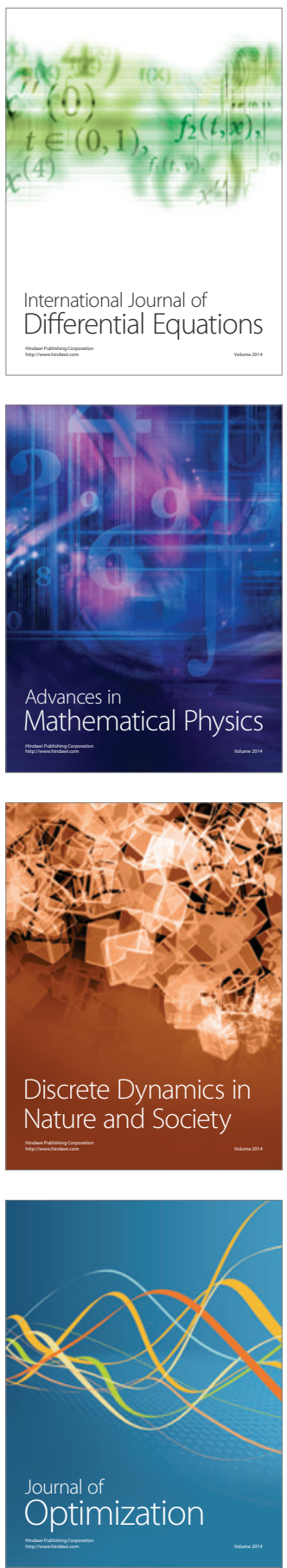